\title{
Шумовые свойства и параметры сигналов в головной части канала считывающей электроники для кремниевых детекторов
}

А. Воронин, К. $\Phi^{-}$М. н. ${ }^{1}$

УДК 539.1.075 | ВАК 05.27.01

\begin{abstract}
При выборе схемотехнического решения для считывающей электроники, используемой в детектирующих системах на основе кремниевых детекторов, необходимо учитывать шумовые свойства измерительного канала как определяющего фактора при измерениях характеристик импульсов в канале. Связано это с тем, что кремниевый детектор - твердотельный аналог газовых ионизационных камер, но, в отличие от последних, не имеюший внутреннего усиления. Сигнал детектора для регистрируемых однозарядных частиц с энергией, эквивалентной энергии одной минимально-ионизирующей частицы (1 м.и.ч.), зависит от толщины детектора и составляет несколько фКл. При этом отношение сигнал/шум редко превышает величину в пределах 2-15. В статье рассмотрены шумовые свойства кремниевого детектора, источники шумов и параметры сигналов головной части считывающей электроники (СЭ), даны рекомендации по реализации схемных решений для головного каскада СЭ.
\end{abstract}

уммарная мощность или напряжение шумов измерительного канала складывается из нескольких составляющих: шума собственно детектора, шумов СЭ и шума элементов, соединяющих детектор со считывающей электроникой. Различные варианты структуры каналов СЭ описаны в [1]. Шумовые свойства СЭ определяют параметры головного каскада, содержащего в общем случае зарядочувствительный усилитель (зчу), который является оптимальным решением для кремниевого детектора [2], и усилители-формирователи (шейперы). следует отметить, что для определения общего шума измерительного канала необходимо рассматривать общую схему детектора и СЭ, поскольку параметры как детектора, так головного каскада СЭ влияют на коэффициенты передачи шумового напряжения или тока отдельных источников шумов, расположенных в разных точках схемы, на суммарный шум выхода 34У. Рассмотрим источники шума детектора, элементов, соединяющих его с СЭ, и головного каскада Сэ.

\section{ШУМОВЫЕ СВОЙСТВА КРЕМНИЕВОГО ДЕТЕКТОРА}

Поскольку подключение кремниевого детектора идентично подключению диода с обратным смещением,

Научно-исследовательский институт ядерной физики

им. Д. В. Скобельцина МГУ им. М.В. Ломоносова,

voronin@silab.sinp.msu.ru. очевидно, что через детектор протекает темновой ток $\mathrm{I}_{0}$, создающий дробовой шумовой ток, спектральная плотность $S_{\text {ID }}$ Которого равна:

$$
S_{I D}=2 \ldots 2,67 e I_{0}
$$

где $\boldsymbol{e}$ - заряд электрона

Для необлученного детектора ток $\mathrm{I}_{0}$ составляет для одного сегмента детектора несколько нА и менее, и в этом случае дробовой шум детектора можно не принимать во внимание. По мере накопления дозы облучения ток $\mathrm{I}_{0}$ может достигать значений в несколько мкА и более, а шум детектора становится сопоставимым с другими составляющими общего шума.

Кроме широкополосного дробового шума, характерного для любого кремниевого детектора, могут возникать низкочастотные импульсные шумы в диапазоне частот 10-100 кГц. Эти шумы могут носить также мерцающий характер.

Для детекторов проекта ZEUS-HES выявлено три причины импульсного шума.

Первая связана с началом лавинного пробоя в детекторе (рис. 1) и зависит от напряжения питания детектора, при этом ток детектора возрастает. Другим источником импульсных шумов в детекторе служит инжекция носителей (электронов / дырок) из объема кремния в окисел на его поверхности у краев активной области детектора (рис. 2). В зависимости от геометрии активной области 


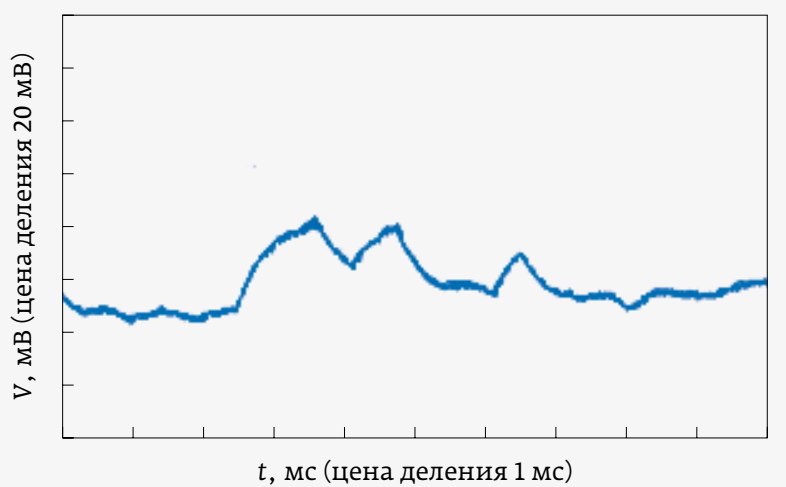

Рис. 1. Импульсный шум, соответствующий началу лавинного пробоя в детекторе

детектора, системы охранных колец и качества проведения фотолитографий инжекция может проявляться при различных напряжениях, приложенных к детектору, но корреляции шумов и величины тока в случае инжекции не наблюдается. Отметим, что импульсные шумы в случае инжекции пропадают при понижении напряжения на детекторе.

Низкочастотный шум детекторов (рис. 3), связанный с различными дефектами структуры детектора, в частности сколами краев детектора при резке или монтаже, сосредоточен в диапазоне частот 0,1-10 кГц. Воздействие такого шума на зчу аналогично сигналу детектора. Кроме того, иногда этот вид шумов наблюдается при вибрации кремниевого детектора.

Низкочастотные шумы могут быть эффективно подавлены шейпером с двойным дифференцированием, который отличается более высокой крутизной передаточной характеристики в диапазоне низких частот

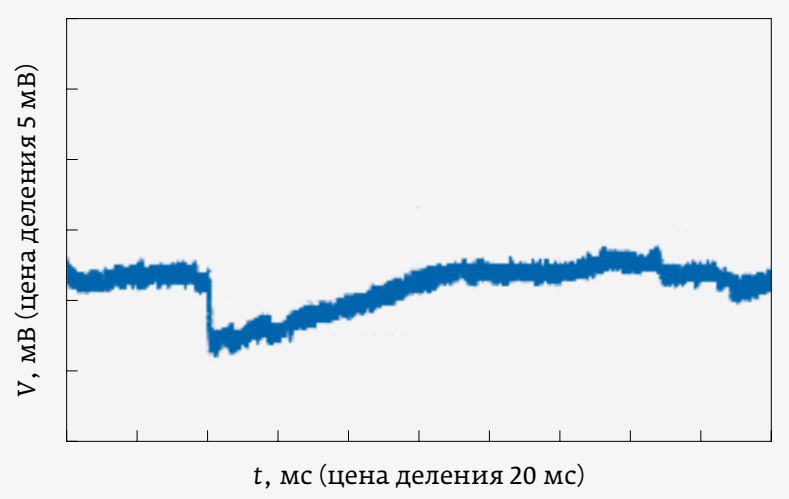

Рис. 3. Низкочастотный шум детекторов, связанный с различными дефектами структуры детектора

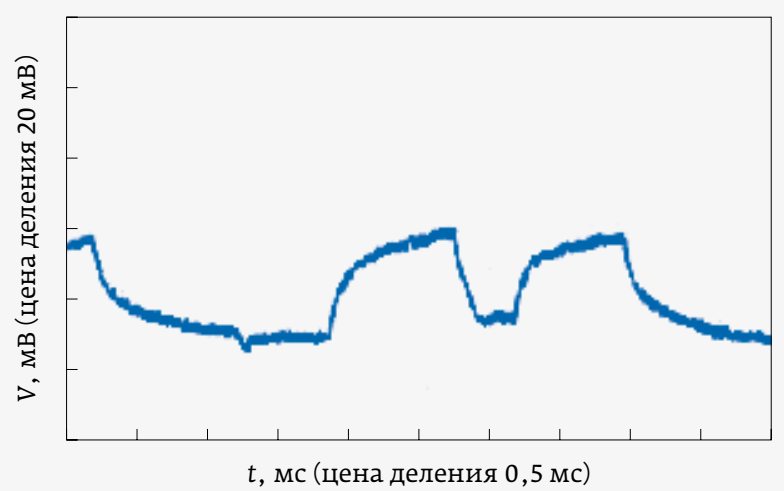

Рис. 2. Импульсный шум при инжекции носителей в окисел на поверхности детектора

(12 дБ / октава) по сравнению со шейпером с одинарным дифференцированием.

Если в детекторе возникает общий для нескольких сегментов дополнительный источник шума (например, локальный пробой детектора), то этот источник через межстриповые емкости будет воздействовать на отдельные сегменты детектора. Возможен случай, когда на одном сегменте детектора возникнут шумовые составляющие, коррелирующие между собой и создающие мультипликативную составляющую. Для двух корреляционных составляющих $P_{n 1}$ и $P_{n 2}$ суммарная мощность шума $P_{n}$ будет равна [3]:

$$
P_{n}=P_{n 1}+P_{n 2}+2 A P_{n 1} P_{n 2}
$$

где $A$ - коэффициент корреляции, изменяющийся от -1 до 1 в зависимости от разности фаз составляющих $\mathrm{P}_{n 1}$ и $\mathrm{P}_{n 2}$.

В этом случае говорят о корреляционном шуме детектора. Корреляционный шум может возникнуть также в системе "детектор - сЭ».

\section{ИСТОЧНИКИ ШУМА СЭ И ЭЛЕМЕНТОВ, СОЕДИНЯЮЩИХ ДЕТЕКТОР СО СЧИТЫВАЮЩЕЙ ЭЛЕКТРОНИКОЙ}

В современных технологических условиях изготовления СЭ основным источником шума в канале служит широкополосный тепловой (белый) шум сопротивления канала МОП-транзистора или активного сопротивления базы для биполярного транзистора, спектральная плот-

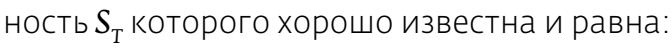

$$
S_{\mathrm{T}}=4 \mathrm{kTR}_{n}
$$

где $\mathrm{R}_{n}$ - эквивалентное шумовое сопротивление электронного прибора, $k$-постоянная Больцмана, T-температура. 


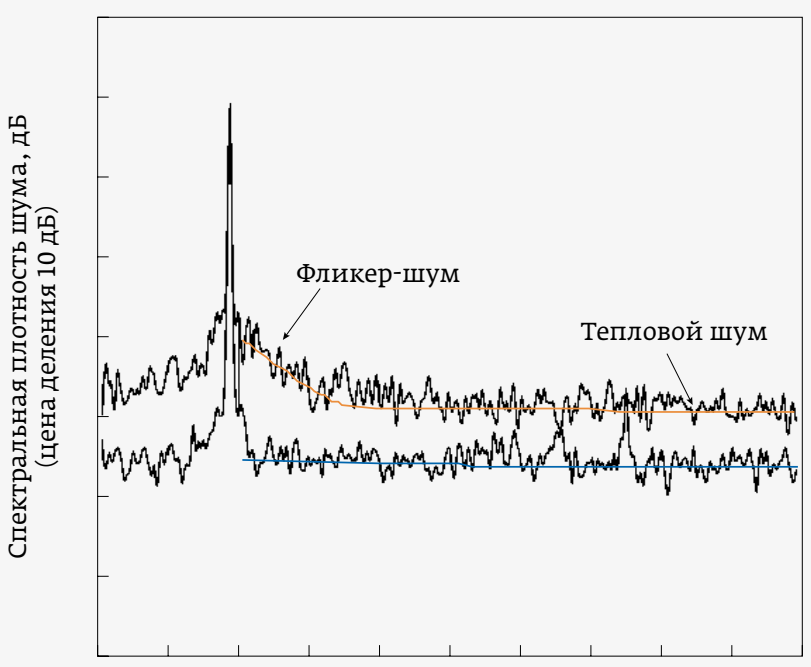

Частота, кГц (цена деления 8,5 кГц)

Рис. 4. Спектральная плотность шума микросхемы CR-1 (тепловой и фликер-шумы)

Этот шум принято называть последовательным, поскольку источник подключен последовательно с входом усилителя.

Последовательный шум входа СЭ имеет также составляющую спектральной плотности $S_{f}$ вида $S_{f}=A / f$, зависящую от частоты $f(A-$ коэффициент, определяемый технологией и типом прибора), или фликер-шум (рис. 4). Известная эмпирическая формула спектральной плотности $S_{f}$ фликер-шума для МОП-транзистора имеет вид:

$$
S_{f}=\left(K_{f} / C_{o x}^{2} W L\right) / f ; C_{o x}=\varepsilon_{o x} \varepsilon_{0} / t_{o x}
$$

где $\mathrm{K}_{f}$ - эмпирическая константа для конкретного технологического процесса (отражена в SPICE-моделях для этого процесса), $\mathrm{K}_{f} \approx(1-40) \cdot 10^{-28}$ Кл / м² для р- и п-канальных МОП-транзисторов соответственно; W и L- ширина и длина канала транзистора; $C_{o x}$ - удельная емкость подзатворного диэлектрика; $\varepsilon_{\text {ох }}$ - диэлектрическая постоянная подзатворного диэлектрика, $t_{\text {ох }}$ - толщина подзатворного диэлектрика.

Отметим, что в современных технологиях фликершум значительно снижен и его следует учитывать при постоянной времени шейпера, равной единицам-десяткам микросекунд.

Для шумов цепи обратной связи зчу свойственна тепловая составляющая (3), и, если цепь обратной связи имеет транзисторные структуры, может возникнуть составляющая A/f. Эти шумы принято называть параллельным шумом, поскольку они подключены параллельно входу усилителя.

Для СЭ стриповых детекторов характерны перекрестные шумы и помехи, которые через межстриповые емкости и другие элементы эквивалентной электрической схемы воздействуют на исследуемый канал.

К указанным источникам шумов в измерительном канале можно добавить шумы сопротивления смещения детектора, сопротивления токопроводящего клея, проволочных соединений микросварки, проводников на поверхности детектора, которые носят в основном тепловой характер (3), а также шумы источников питания.

На основе приведенных сведений можно составить эквивалентную схему головной части СЭ с подключенным детектором и источниками шумов.

\section{ЭКВИВАЛЕНТНАЯ СХЕМА ГОЛОВНОЙ ЧАСТИ СЭ С ИСТОЧНИКАМИ ШУМОВ}

Эквивалентная схема головной части измерительного канала СЭ с источниками шумов и основных элементов схемы, существенно влияющих на суммарный шум канала, представлена на рис. 5. На схеме не показаны перекрестные помехи от сигналов соседних каналов. Перечислим элементы, которые входят в состав схемы:

- $34 У$ с эквивалентной суммарной емкостью $C_{\Sigma}=C_{d}$ [4], содержащей все эквивалентные емкостные составляющие, подключенные к входу з4у, основная часть которой-емкость детектора (входная емкость собственно зчу не учитывается ввиду ее малости по сравнению $с C_{d}$ в современных технологических условиях); источник последовательного шума $e_{a}$ 34У, в который входят тепловой шум (3) и фликершум (4); цепь обратной связи $34 У ~ \mathrm{R}_{f b} \mathrm{C}_{f b}$ с источником шума $e_{f b}(3)$;

- кремниевый детектор, который содержит источник шумового дробового тока $S_{\text {ID }}(1)$ и других шумов детектора (импульсных - рис. 1-3), источник шумового корреляционного тока $\mathrm{P}_{n}(2) ; \boldsymbol{e}_{d}$ - суммарный шум детектора. Емкость и сопротивление на обратную сторону $\mathrm{C}_{b}$ и $\mathrm{R}_{b}$; межстриповая емкость $\mathrm{C}_{\text {is, }}$ к которой подключен последовательный источник шума соседнего канала $e_{a 1}$. Для второго соседнего канала схема подключения источника шума $e_{a 2}$ аналогична: $e_{a 1}=e_{a 2}=e(3)$;

- сопротивление цепи источника смещения $\mathrm{R}_{b l}$ и источник $e_{b l}$ (3) для стрипового детектора, а также $\mathrm{R}_{b l}$ и источник $e_{b l}$ для падового детектора;

- элементы, связанные с технологией крепления детектора к основанию (подложке): $\mathrm{R}_{b s}$ и $\mathrm{C}_{b s}$ с источником шума $e_{b s}(3)$;

- элементы, связанные с технологией соединения детектора с входом зчу: $\mathrm{R}_{\mathrm{k}}$ с источником шума $e_{\mathrm{k}}(3)$; 

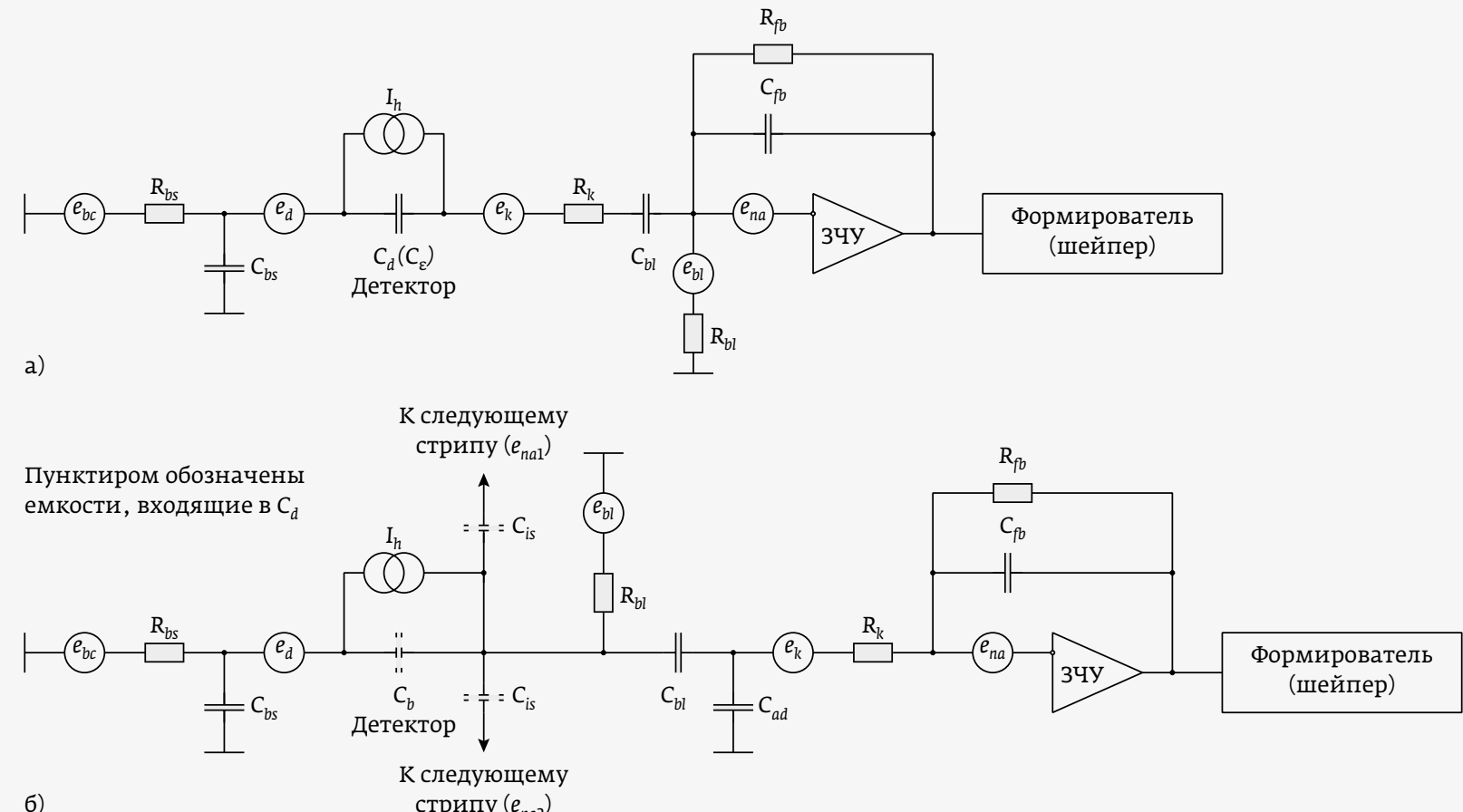

б)

стрипу $\left(e_{\text {па2 }}\right)$

Рис. 5. Эквивалентная схема головной части измерительного канала Сэ с источниками шумов и основными элементами схемы: а - для падового детектора; 6 - для стрипового детектора

- блокировочная емкость $C_{b l}$, расположенная на стриповом детекторе, или внешняя блокированная емкость, величину которой можно выбрать много больше $C_{d}$ и ее можно не учитывать;

- формирователь (шейпер). Разнообразные типы шейперов подробно описаны в [3]

В соответствии со схемой (см. рис. 5) источники шумов расположены в разных точках схемы и, соответственно, коэффициенты передачи шума на выход ЗчУ различаются. Это означает, что суммарное напряжение шума в системе "детектор - Зчу» формируется на выходе зчу. Оценить суммарный шум, приведенный к входу зчу (в Кл), можно через коэффициент передачи зчу, равный приблизительно $1 / C_{f b}$. Следует отметить, что выходное напряжение зчу и падение напряжения на цепи обратной связи зчУ равны

Шум фильтруется шейпером. Нормируя напряжение шума на выходе шейпера посредством максимального значения коэффициента передачи для шейпера и используя процедуру для зчу, можно получить приведенный шум к входу зчу (в Кл), взвешенный в полосе частот шейпера, который определяет приведенный к входу шум измерительного канала.

\section{ПРЕОБРАЗОВАНИЕ ВХОДНЫХ ШУМОВ ИЗМЕРИТЕЛЬНОГО КАНАЛА В ШУМОВОЕ НАПРЯЖЕНИЕ ГОЛОВНОЙ ЧАСТИ КАНАЛА Дробовой шум $\mathrm{I}_{n}$}

Шумовые токи детектора (включая ток входного транзистора) (1) протекают через цепь обратной связи зчУ и на его сопротивлении $\mathrm{Z}_{f b}\left(\tau_{f b}=\mathrm{R}_{f b} \mathrm{C}_{f b}\right)$, равном

$$
Z_{f b}=\frac{R_{f b}}{1+s \cdot \tau_{f b}},
$$

создают напряжение $U_{i n}$, которое на выходе шейпера равно:

$$
\begin{aligned}
U_{i n} & =\sqrt{S_{I D} \int_{0}^{\infty}\left|Z_{f b}\right|^{2} \cdot \frac{k_{s h}^{2}(2 \pi f)}{1+\left(2 \pi f \tau_{p 1}\right)^{2}} d f} \cong \\
& \cong \sqrt{S_{I D} \cdot R_{f b}^{2} \int_{0}^{\infty} \frac{k_{s h}^{2}(2 \pi f)}{1+\left(2 \pi f \tau_{f b}\right)^{2}} d f},
\end{aligned}
$$

где $k_{\text {sh }}$ - нормированный коэффициент передачи шейпера, если считается, что полюс зчу $\tau_{p 1}$ далеко отстоит от начала полосы шейпера, и используется понятие шумовой полосы [5], которая для зчу равна: 


$$
N_{c s a}=\frac{\tau_{f b}^{2}}{\tau_{f b}^{2}-\tau_{p 1}^{2}} \cdot\left(\frac{1}{4 \tau_{p 1}}-\frac{1}{4 \tau_{f b}}\right) \cdot \cong \frac{1}{4 \tau_{p 1}}-\frac{1}{4 \tau_{f b}} .
$$

Выражение (6) можно представить без учета $\tau_{p 1}$ в виде:

$$
U_{i n_{-} c s a} \cong \sqrt{S_{I D} \cdot R_{f b}^{2} / 4 R_{f b} C_{f b}}
$$

Рассчитанная величина дробового шума, приведенная к входу зчУ, для канала проекта HES-ZEUS составила $0,24 \mathrm{fC} \cdot \sqrt{\mathrm{I}}$ [мкA], а измеренная $-0,22 \mathrm{fC} \cdot \sqrt{\mathrm{I}}[\mathrm{Mк} A]$, где I- ток утечки детектора и входа зчу

\section{Шум источника смещения диода $\boldsymbol{e}_{b l}$}

Оптимальной схемой смещения можно считать схему, когда параметры фильтров питания выбраны таким образом, что они подавляют перекрестные и другие помехи во всей полосе частот и не вносят собственных шумов источника питания в общий шум канала, а также шумы от $R_{b l}$.

Коэффициент передачи для шумов $\mathrm{R}_{b l}$ равен:

$$
k_{b l}(s)=\frac{R_{f b}}{R_{b l}} \cdot \frac{1}{1+R_{f b} \cdot C_{f b} \cdot s} \cdot \frac{R_{b l} \cdot C_{b l} \cdot s}{1+R_{b l} \cdot C_{b l} \cdot s} \cdot \frac{1}{1+\tau_{p 1} \cdot s} \cdot k_{s h}(s) .
$$

Выражение (9) можно упростить, поскольку $\tau_{p 1}<<\mathrm{R}_{f b} \mathrm{C}_{f b}$ и $\mathrm{C}_{b} \gg \mathrm{C}_{d}$. Очевидно, что $\mathrm{R}_{b l}$ лучше увеличивать, снижая тем самым усиление шума. В дополнение к фильтрации шумов шейпером 34 является фильтром нижних частот этого вида шумов. Шумовое напряжение на выходе 34 у $U_{\text {nb_csa }}$ и шейпера $U_{\text {nb_sh }}$ соответственно равны:

$$
\begin{gathered}
U_{n b_{-} s c a} \cong \frac{R_{f b}}{\sqrt{R_{b l}}} \sqrt{k \cdot T \cdot\left(\frac{1}{R_{f b} C_{f b}}-\frac{1}{R_{b l} C_{b l}}\right)}, \\
U_{n b \_s h}=\sqrt{\frac{4 k T R_{f b}^{2}}{R_{b l}} \int_{0}^{\infty} \frac{\left(2 \pi f R_{b l} C_{b l}\right)^{2} \cdot k_{s h}^{2}(2 \pi f)}{\left(1+\left(2 \pi f R_{b l} C_{b l}\right)^{2}\right) \cdot\left(1+\left(2 \pi f R_{f b} C_{f b}\right)^{2}\right)^{2}} d f .}
\end{gathered}
$$

Величина $\mathrm{R}_{b l}$ может оказывать влияние также на коэффициент передачи последовательного шума $\boldsymbol{e}_{a}$

\section{Параллельный шум схемы разряда емкости обратной связи ЗЧУ $\boldsymbol{e}_{f b}$}

Без учета полюса зчУ при шумовой полосе цепи обратной связи $1 / 4 \mathrm{R}_{f b} \mathrm{C}_{f b}$ напряжение шумов на выходе 34 У $\mathrm{U}_{\mathrm{nfb}}$ сsа $\mathrm{PaBHO}$

$$
U_{n f b_{-} s s a} \cong \sqrt{k T / C_{f b}} .
$$

Этот вид шумов часто называют kT / C-шумом, то есть при отсутствии формирователя шум в канале не зависит от величины резистора обратной связи $\mathrm{R}_{f b}$ и снижается при увеличении $C_{f b}$. Введение шейпера $\left(k_{s h}\right)$ изменяет поведение шума на его выходе и осуществляет фильтрацию параллельного шума:

$$
U_{n f b_{-} s h}^{2}=4 \mathrm{kTR}_{f b} \int_{0}^{\infty} \frac{k_{s h}^{2}(2 \pi f)}{1+\left(2 \pi f \mathrm{R}_{f b} C_{f b}\right)^{2}} d f .
$$

В качестве примера приведем фильтрацию CR-RC-шейпером с постоянной времени $\tau_{\text {sh }}=\mathrm{CR}$, в этом случае напряжение шума на выходе шейпера равно:

$$
U_{n f b_{-} s h}=\frac{\sqrt{2 k T R_{f b} \tau_{s h}}}{\tau_{s h}+R_{f b} C_{f b}} .
$$

Нормированный график шумов показан на рис. 6.

Фильтрация CR-RC-шейпером приводит к появлению зависимости шумов канала от термального шума $\mathrm{R}_{f b}$, при которой шум равен нулю для $\mathrm{R}_{f b}=0, \mathrm{R}_{f b} \rightarrow \infty$ и максимум, равный $\frac{\sqrt{2}}{2} \cdot \sqrt{\frac{k T}{C_{f b}}}=\frac{\sqrt{2}}{2} \cdot e_{n f b_{-} c s a}$ для $\mathrm{R}_{f b}=\tau_{s h} / C_{f b}$, то есть при условии, когда постоянные времени зчу и шейпера равны, а коэффициент передачи максимален. При сопротивлении $\mathrm{R}_{f b}$, равном нулю, шум (1) равен нулю. Увеличение $\mathrm{R}_{f b}$ приводит к возрастанию шума и приближению полосы ЗЧУ по частоте к полосе шейпера. По мере роста $\mathrm{R}_{f b}$ полосы частот шейпера и зчУ расходятся по частоте и начинается снижение шума, поэтому для уменьшения параллельного шума постоянную времени спада зчу

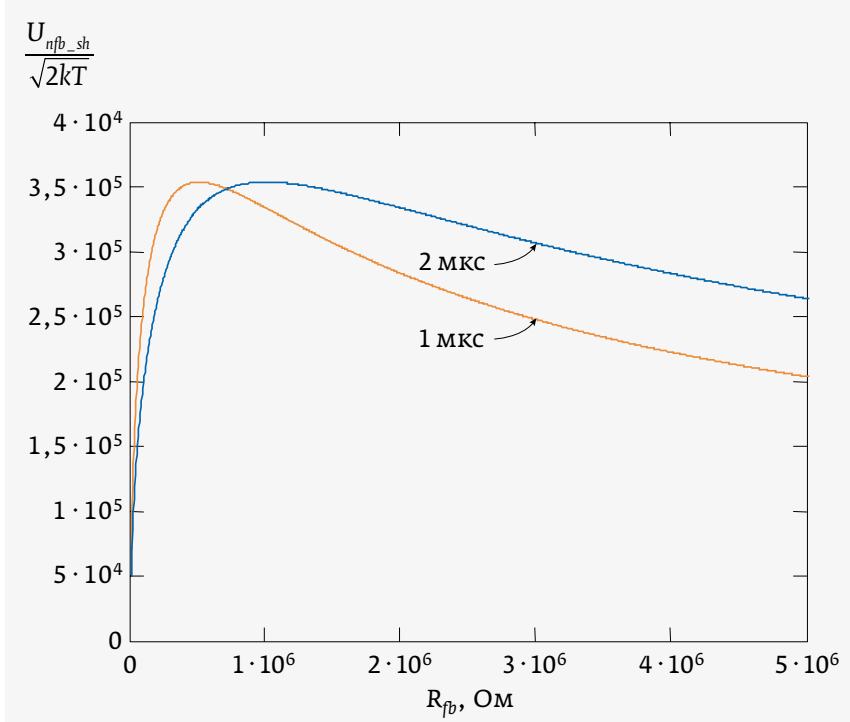

Рис. 6. Параллельный шум на выходе шейпера для $\tau_{\text {sh }}=1$ и 2 мкс 
стараются по возможности увеличить. Применение компенсатора полюса 3ЧУ, когда выходной отклик формирователя не зависит от $\tau_{f b}$, позволяет использовать сравнительно низкоомный резистор $\mathrm{R}_{f b}$. При этом параллельный шум может быть также снижен. Результаты, представленные в этом разделе, согласуются с экспериментальными данными, полученными при измерении микросхемы CR-1 проекта ATIC.

\section{Последовательный шум $\boldsymbol{e}_{a}$}

Последовательный шум усилителя $U_{n a}$ в большинстве случаев - основная шумовая составляющая общего шума канала - содержит две части: широкополосную (3), где $\mathrm{R}_{\mathrm{n}}=1 / \mathrm{S}\left(\mathrm{R}_{\mathrm{k}}\right.$ - активное сопротивление канала транзистора, $\boldsymbol{S}$ - крутизна характеристики транзистора в рабочей точке) и низкочастотную часть - фликер-шум (4):

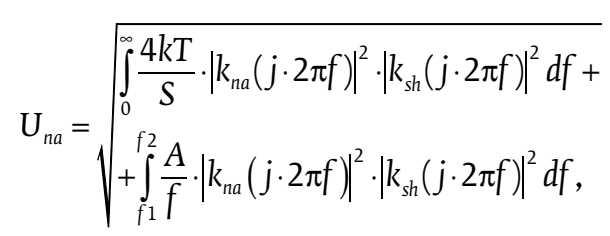

где $k_{\text {nа }}$ - коэффициент передачи зчу, частоты $f_{1}$ и $f_{2}$ выбираются из соображений, когда фликер-шум (4) превышает широкополосную спектральную плотность (3). Исходя из теории шестиполюсников [5] и схемы рис. 5 (без учета сопротивления контактов, $\mathrm{R}_{k}=\mathrm{R}_{\mathrm{bs}}=0$ ) получим модуль коэффициента передачи $34 \mathrm{y} \mathrm{k}_{n a}\left(\mathrm{C}_{b l}>>\mathrm{C}_{d}\right)$ :

$$
\begin{gathered}
\left|k_{n a}\right| \cong \sqrt{\frac{\left(1+R_{f b} / R_{b l}\right)^{2}+\left(2 \pi f R_{f b} C_{f b}+2 \pi f R_{f b} C_{d}\right)^{2}}{1+\left(2 \pi f R_{f b} C_{f b}\right)^{2}} \times} \\
\times \frac{1}{\sqrt{1+\left(2 \pi f \tau_{p 1}\right)^{2}}} .
\end{gathered}
$$

и для высокоомного $\mathrm{R}_{b l}$ :

$$
\left|k_{n a}\right| \approx\left(1+\frac{C_{d}}{C_{f b}} \cdot \frac{2 \pi f R_{f b} C_{f b}}{\sqrt{1+\left(2 \pi f R_{f b} C_{f b}\right)^{2}}}\right) \cdot \frac{1}{\sqrt{1+\left(2 \pi f \tau_{p 1}\right)^{2}}} .
$$

Проиллюстрируем полученный результат с учетом итогов испытаний, проведенных в ходе работ над экспериментальными прототипами детекторов и $34 у$ (рис. 7). Для высокоомного $\mathrm{R}_{b l}$ зчУ всегда представляет собой широкополосный фильтр с частотами среза $1 / 2 \pi \tau_{f b} n 1 / 2 \pi \tau_{p 1}$ и оценкой коэффициента усиления в полосе фильтра $\cong 1+C_{d} / C_{f b}$. Эта оценка обычно берется для вычисления $U_{n a}$. Кривая 2 соответствует случаю, когда $C_{d}=C_{d}>C_{f b}$; для $\mathrm{C}_{d}$, близком к $\mathrm{C}_{f b}$, усиление падает (кривая 5). При постепенном снижении $R_{b l}$ поведение усиления

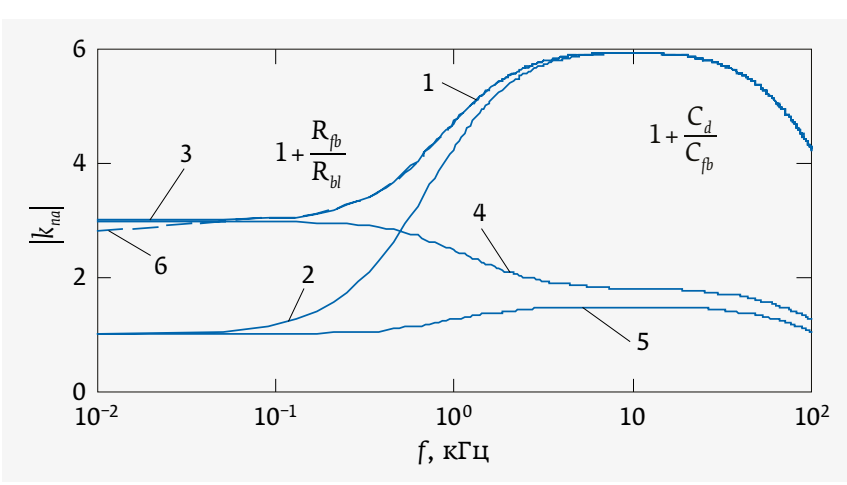

Рис. 7. Коэффициенты передачи шумов $U_{\text {nа, }}$ для различных значений элементов схемы рис. 5

в высокочастотной области для $\mathrm{R}_{b l} \mathrm{C}_{d}>\mathrm{R}_{f b} \mathrm{C}_{f b}$ (кривая 3) напоминает поведение усилителя для высокоомного $\mathrm{R}_{b l}$. Однако усиление низкочастотного шума увеличено и составляет $\cong 1+\mathrm{R}_{f b} / \mathrm{R}_{b l}$. В случае $\mathrm{R}_{b l} \mathrm{C}_{d}<\mathrm{R}_{f b} \mathrm{C}_{f b}$ (кривая 4) поведение усиления шума от частоты напоминает фильтр нижних частот с теми же значениями усиления в низкочастотной области $\cong 1+\mathrm{R}_{f b} / \mathrm{R}_{b l}$, а в высокочастотной - наблюдается сложение кривой фильтра нижних частот и кривой полосового фильтра с преобладанием влияния низкочастотного фильтра.

На сверхнизких частотах, особенно для стриповых детекторов, когда $C_{b l}$ не может быть достаточно большой из-за технологических ограничений, сказывается влияние $C_{b l}$ (пунктир 6 и кривая 1, совпадающая в ВЧ-области с кривой 3, на рис. 7):

$$
\mid k_{n a}=\sqrt{\frac{\left[1-a \omega^{2} \tau_{b} R_{b l} C_{d}\right]+\left[\omega \tau_{f b}+a \omega \tau_{b}\right]^{2}+}{+\left(\omega \tau_{b}\right)^{2}\left[\left(1+\left(\omega \tau_{f b}\right)^{2}\right)+2 a\left(1+\omega^{2} R_{b l} C_{d} \tau_{f b}\right)\right]}}[.
$$

где $a=\mathrm{R}_{f b} / \mathrm{R}_{b l}, \tau_{b}=\mathrm{R}_{b l} \mathrm{C}_{b l}, \omega=2 \pi f, \mathrm{C}_{b l} \gg \mathrm{C}_{d}$.

Если сопротивления контактов детектора $R_{b s}$ и $R_{k}$ (см. рис. 5) не равны нулю, они могут оказывать существенное влияние на передачу последовательного шума на выход зчу. В операторной форме для падовых детекторов коэффициент передачи $k_{n a}(s)$ последовательного шума $e_{a}$ выглядит:

$$
k_{n a}(s)=\left(\begin{array}{c}
1+\frac{s \cdot R_{f b} C_{d}}{\left(1+s \cdot R_{f b} C_{f b}\right)}+ \\
s \cdot R_{f b} C_{d} \cdot\left(1+s \cdot R_{b s} C_{b s}\right) \\
+\frac{1}{\left[s \cdot R_{b s} C_{d}+\left(1+R_{k} C_{d}\right) \cdot\left(1+s \cdot R_{b s} C_{b s}\right)\right] \cdot\left(1+s \cdot R_{f b} C_{f b}\right)}
\end{array}\right) \times
$$




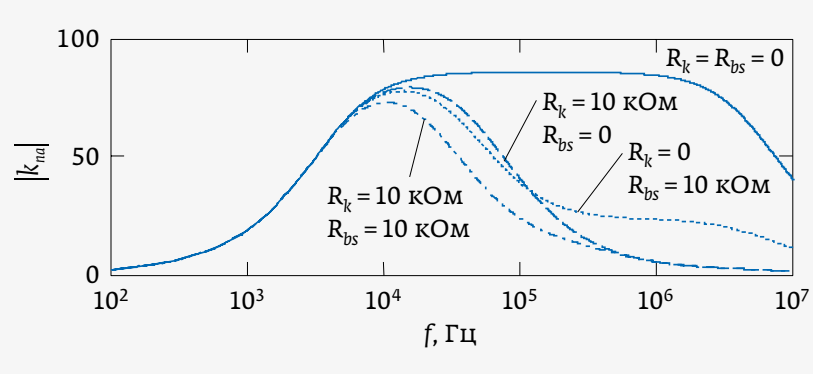

Рис. 8. Амплитудно-частотные характеристики Зчу для шума $e_{a}$ в зависимости от сопротивлений $\mathrm{R}_{\mathrm{k}}$ и $\mathrm{R}_{b s}$ (для $\left.f=0, k_{\text {na }}=1(19)\right)$

Типичные графики модуля коэффициента передачи последовательного шума на выходе $34 у\left|k_{\text {na }}(2 \pi f)\right|$ для падовых детекторов (АЧХ) приведены на рис. 8. Параметры $C_{d}=300 \quad \Pi \Phi, C_{i n}=6 \quad \sqcap \Phi, C_{f b}=3,6 \sqcap \Phi, C_{b s}=100 \quad \Pi \Phi$, $\mathrm{R}_{f b}=10$ МОм выбраны близкими аналогичным параметрам схемы проекта HES. В случае, когда постоянные времени $\mathrm{R}_{b s} \mathrm{C}_{b s}$ и $\mathrm{R}_{k} \mathrm{C}_{d}$ меньше, чем $\tau_{f b}$, полоса 34 у уменьшается. Максимум коэффициента передачи не превышает или равен $\cong 1+C_{d} / C_{f b}$. Но, как будет показано ниже, при этом может уменьшаться амплитуда сигнала и ухудшаться отношение сигнал / шум.

Напряжение последовательного шума $U_{\text {nа }}$ на выходе зчу без формирователя в зависимости от сопротивлений контактов приведено на рис. 9. Как видно из графиков, при малых значениях $\mathrm{R}_{k}$ и $\mathrm{R}_{b s}$ их влияние на величину $\mathrm{U}_{n a}$ примерно одинаково.

При достаточно больших значениях $\mathrm{R}_{k}$ либо $\mathrm{R}_{b s}$ максимальный коэффициент передачи падает, и для $\mathrm{R}_{k} \rightarrow \infty$ шум становится равным шуму усилителя с единичным усилением. Для $\mathrm{R}_{k}=0$ и $\mathrm{R}_{b s} \rightarrow \infty$ усиление шума $e_{n a}$ стремится К $1+C_{s} / C_{f b}$, где $C_{s}=\left(C_{d} \cdot C_{b s}\right) /\left(C_{d}+C_{b s}\right)$.

В стриповом детекторе последовательный шум - основная составляющая, при этом шум соседних каналов

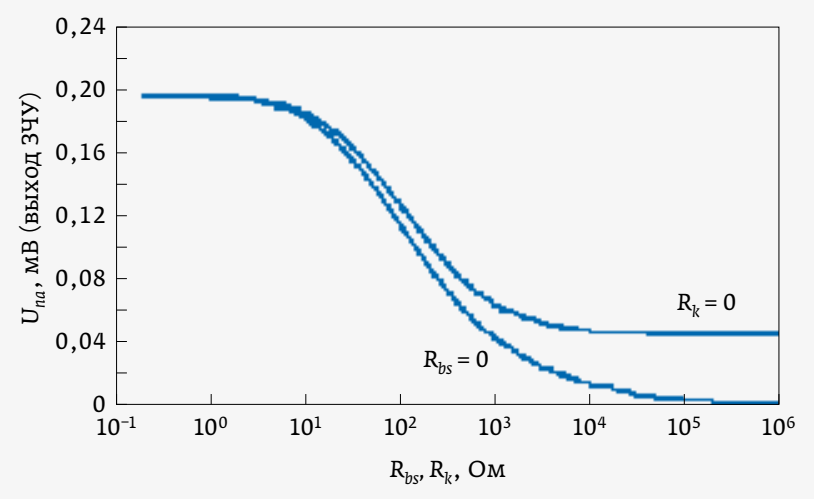

Рис. 9. Последовательный шум Зчу без формирователя в зависимости от $\mathrm{R}_{b s}$ и $\mathrm{R}_{k}$ через межстриповые емкости $C_{\text {is }}$ (см. рис. 5) попадает на вход 34У, пройдя через делитель $C_{i s,} C_{b}\left(C_{b} \approx 0,1 C_{i s}\right)$ [3]. К тому же шум соседнего канала делится между соседними стрипами, поэтому с учетом влияния двух соседних стрипов можно считать, что последовательный шум канала в стриповом детекторе на $\approx 20 \%$ выше шума входного транзистора отдельно взятого канала. В данном случае влияние шумов соседних каналов уже не учитывается.

\section{Шумы контактов детектора $\boldsymbol{e}_{b s} \boldsymbol{n}_{\mathrm{k}}$}

Желательно, чтобы сопротивление контактов детектора $\mathrm{R}_{\mathrm{k}}$ (включает сопротивление проводников, в том числе на поверхности детектора (стрипа) и микросварки) и $\mathrm{R}_{b s}$ (см. рис. 5) было равным нулю, что исключает шумы этих элементов. Однако это не всегда представляется возможным из-за качества токопроводящего клея (наблюдалось в проекте ZEUS-HES), конечного удельного сопротивления проводников и других факторов. Напряжение шума на выходе $34 У U_{n k}$ и $U_{n b k}$ равно:

$$
\begin{gathered}
U_{n k}=\sqrt{4 k R_{k} \cdot \int_{0}^{\infty}\left|k_{k}(j \cdot 2 \pi f)\right|^{2} \cdot\left|k_{s h}(j \cdot 2 \pi f)\right|^{2} d f}, \\
U_{n b s}=\sqrt{4 k T R_{b s} \cdot \int_{0}^{\infty}\left|k_{b s}(j \cdot 2 \pi f)\right|^{2} \cdot\left|k_{s h}(j \cdot 2 \pi f)\right|^{2} d f} .
\end{gathered}
$$

Следует отметить, коэффициенты передачи шумов для падовых детекторов $R_{k}$ и $R_{b s}$ взаимосвязаны, то есть в выражениях для $\mathrm{R}_{k}$ и $\mathrm{R}_{b s}$ представлены обе величины.

В стриповых детекторах, когда $C_{b} \approx 0,1 C_{i s}$, влияние $R_{b s}$ на общий шум мало и можно в приведенных ниже выражениях полагать $\mathrm{R}_{b s}$ равным нулю.

На основе теории шестиполюсников [5] получим модули коэффициентов передачи шумов $k_{b s} n k_{k}$ для $\mathrm{R}_{b s}$ и $\mathrm{R}_{\mathrm{k}}$ для падовых детекторов на выходе 34у:

$$
\begin{aligned}
& \left|k_{b s}(2 \pi f)\right|=\frac{C_{d}}{C_{f b}} \cdot \frac{2 \pi f \tau_{f b}}{\sqrt{1+\left(2 \pi f \tau_{f b}\right)^{2}}} \times \\
& \times \frac{1}{\sqrt{\left(\left(1-(2 \pi f)^{2} \cdot R_{b s} R_{k} C_{b s} C_{d}\right)^{2}+(2 \pi f)^{2}\left(R_{k} C_{d}+R_{b s} C_{d}+R_{b s} C_{b s}\right)^{2}\right.}} \times \\
& \times \frac{1}{\sqrt{1+(2 \pi f)^{2} \cdot \tau_{p 1}^{2}}}, \\
& \left|k_{k}(2 \pi f)\right|=\frac{C_{d}}{C_{f b}} \cdot \frac{2 \pi f \tau_{f b}}{\sqrt{1+\left(2 \pi f \tau_{f b}\right)^{2}}} \times \\
& \times \frac{\sqrt{1+\left(2 \pi f \mathrm{R}_{b s} \mathrm{C}_{b s}\right)^{2}}}{\sqrt{\left(\left(1-(2 \pi f)^{2} \cdot\left(\mathrm{R}_{b s} \mathrm{R}_{k} \mathrm{C}_{b s} \mathrm{C}_{d}\right)^{2}+(2 \pi f)^{2}\left(\mathrm{R}_{k} \mathrm{C}_{d}+\mathrm{R}_{b s} \mathrm{C}_{d}+\mathrm{R}_{b s} \mathrm{C}_{b s}\right)^{2}\right.\right.}} \times \\
& \times \frac{1}{\sqrt{1+(2 \pi f)^{2} \cdot \tau_{p 1}^{2}}} .
\end{aligned}
$$




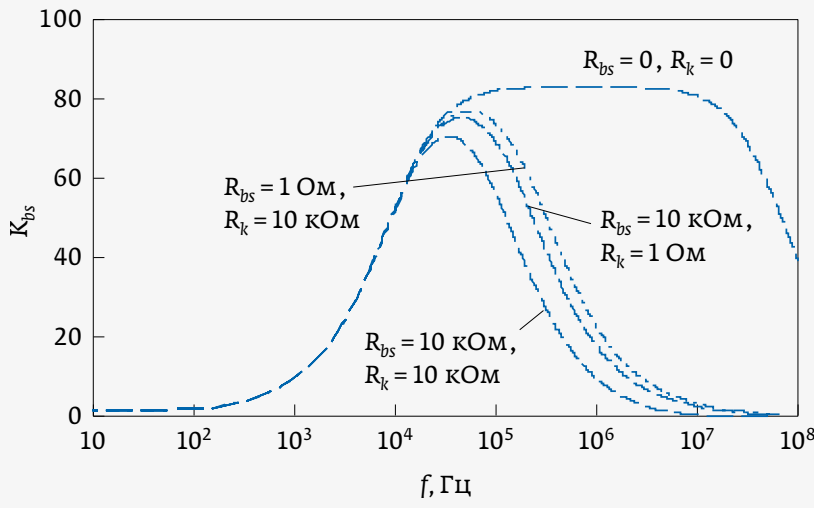

Рис. 10. Амплитудно-частотные характеристики зчу для шумов сопротивления $\mathrm{R}_{b s}$

Графическое изображение выражений (21) и (22) представлено для различных сочетаний $\mathrm{R}_{\mathrm{k}}$ и $\mathrm{R}_{\mathrm{bs}}$ на рис. 10 и 11 со значениями элементов схемы (рис. 5), приведенных в предыдущем разделе для проекта HES.

По мере увеличения $\mathrm{R}_{k}$ и $\mathrm{R}_{b s}$ растут постоянные времени цепей, в которые входят эти резисторы, что, в свою очередь, приводит к сужению полосы пропускания зчу и смещению максимума полосы в низкочастотную область. При этом падает коэффициент усиления в полосе, поскольку зчу начинает работать как усилитель напряжения с частотно-зависимой обратной связью. Некоторое снижение наклона характеристики в высокочастотной области для $k_{k}$ (см. рис. 11) связано с тем, что в (22) в числителе имеется дополнительный член (по сравнению с (21)), который увеличивается по меpe pоста $\mathrm{R}_{b s} \mathrm{C}_{b s}$.

Рассмотрим поведение выходного шума $34 у U_{\text {nbs, }}$ используя выражения (20), (21) в зависимости от $\mathrm{R}_{b s}$ при

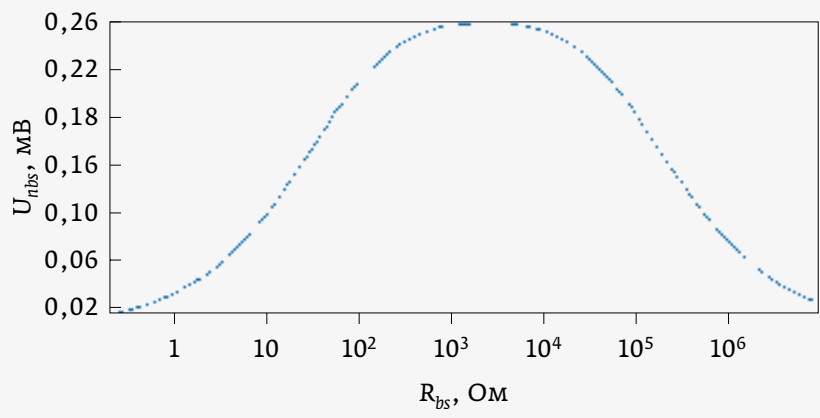

Рис. 12. Шум резистора $R_{b s}$ на выходе ЗЧу при низкоомном $R_{k}=1$ OM

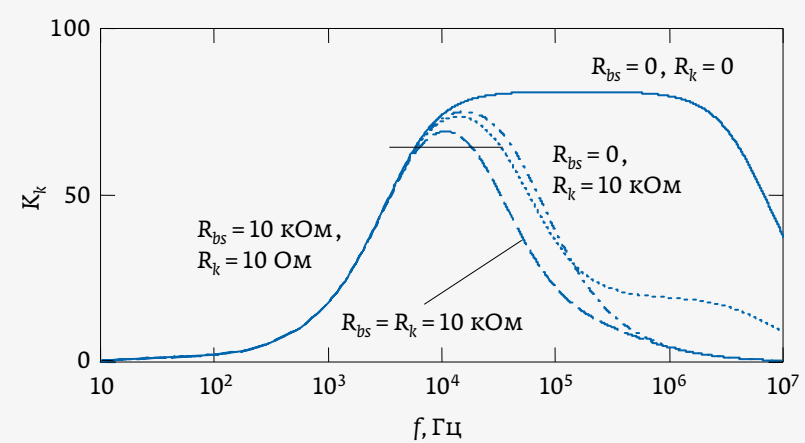

Рис. 11. Амплитудно-частотные характеристики зчу для шумов сопротивления $R_{k}$

малом $\mathrm{R}_{k}=1$ Ом, то есть соединение детектора с входом зчУ считаем низкоомным (рис. 12).

При низких значениях $\mathrm{R}_{b s}$ напряжение его шумов

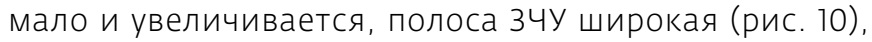
поэтому наблюдается рост шумов. Если полоса частот зчу уменьшается, падает усиление, и величина $U_{n b s}$ снижается. Когда шумы $R_{b s}$ уже достаточно велики, а полоса зчу еще широка, на выходе зчу наблюдается максимум значения напряжения шума. Такое пове-

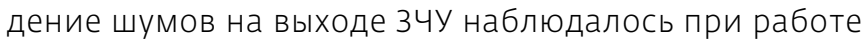
над проектом HES, оно же характерно и для шумов $\mathrm{R}_{k}$ для низкоомного $\mathrm{R}_{\mathrm{bs}}$.

Другое поведение шумов $U_{n k}$ сопротивления $R_{k}$ имеет место, если, например, величина $\mathrm{R}_{k}=100$ Ом (рис. 13).

Пока $\mathrm{R}_{k}$ существенно больше $\mathrm{R}_{b s}$, шумы определяются сопротивлением $\mathrm{R}_{\mathrm{k}}$. Когда эти сопротивления сравнимы, коэффициент передачи для $\boldsymbol{e}_{\text {nk. }}$ падает. При высокоомном

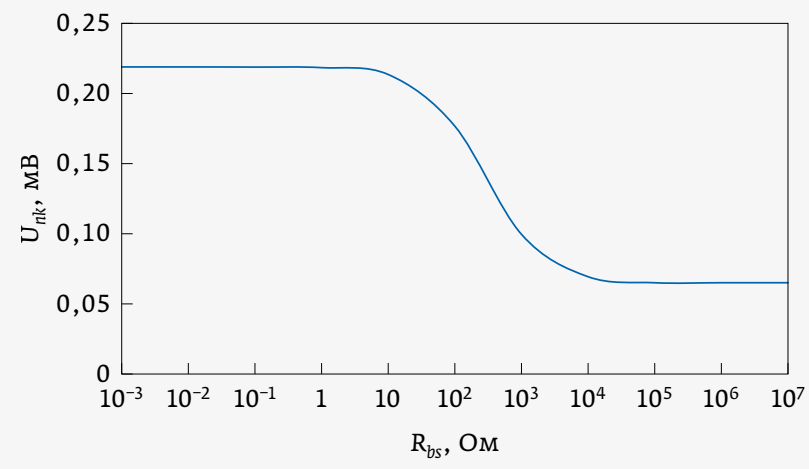

Рис. 13. Шум резистора $R_{k}=100$ Ом для различных $R_{b s}$ 


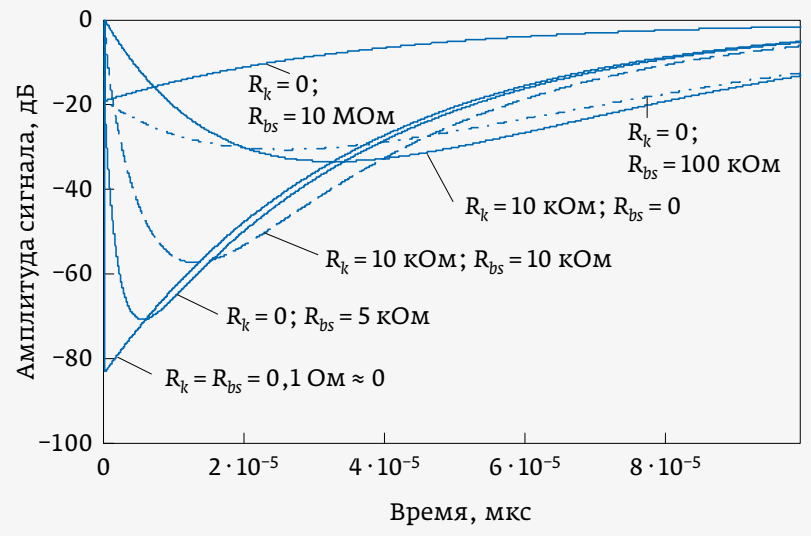

Рис. 14. Форма сигнала на выходе зчу для различных значений $R_{b s}$ и $R_{k}$

$\mathrm{R}_{b s^{\prime}}$ когда им можно пренебречь, коэффициент передачи для $e_{\text {nk. }}$ Определяется в основном емкостями схемы. Напряжение шума при этом на выходе зчУ не зависит от резистора $\mathrm{R}_{\mathrm{bs}}$.

Примеры, основанные на экспериментальных работах над проектом HES, показывают, что минимальный шум, вносимый сопротивлениями $\mathrm{R}_{k}$ и $\mathrm{R}_{b s}$, можно не учитывать, когда их величины не превышают нескольких Ом.

\section{СИГНАЛЫ ГОЛОВНОЙ ЧАСТИ СЭ И ОТНОШЕНИЕ СИГНАЛ / ШУМ}

Качество работы измерительного канала (флуктуации измерений) с кремниевым детектором в первую очередь определяет соотношение сигнал / шум. В предыдущих разделах были рассмотрены шумовые источники канала и коэффициенты передачи шумов этих источников для головной части СЭ. Сейчас речь пойдет о формировании сигнала в головной части СЭ от пролета ионизирующих частиц через детектор с учетом паразитных элементов $R_{k} и R_{b s}$.

Используя входное воздействие $1 / s$ (перепад напряжения), запишем выходное напряжение зчУ в операторной форме:

$$
\begin{gathered}
\mathrm{U}_{c s a}(s)=-\frac{C_{d}}{C_{f b}} \cdot \frac{\tau_{f b}}{1+s \cdot \tau_{f b}} \cdot \frac{1}{1+s \cdot \tau_{p 1}} \times \\
\times \frac{1+s \cdot \tau_{b s}}{1+\left(\tau_{b s}+\tau_{k}+\tau_{d}\right) \cdot s+\tau_{b s} \cdot \tau_{k} \cdot s^{2}},
\end{gathered}
$$

где $\tau_{b s}=R_{b s} C_{b s}, \tau_{k}=R_{k} C_{d}, \tau_{d}=R_{b s} C_{d}$.

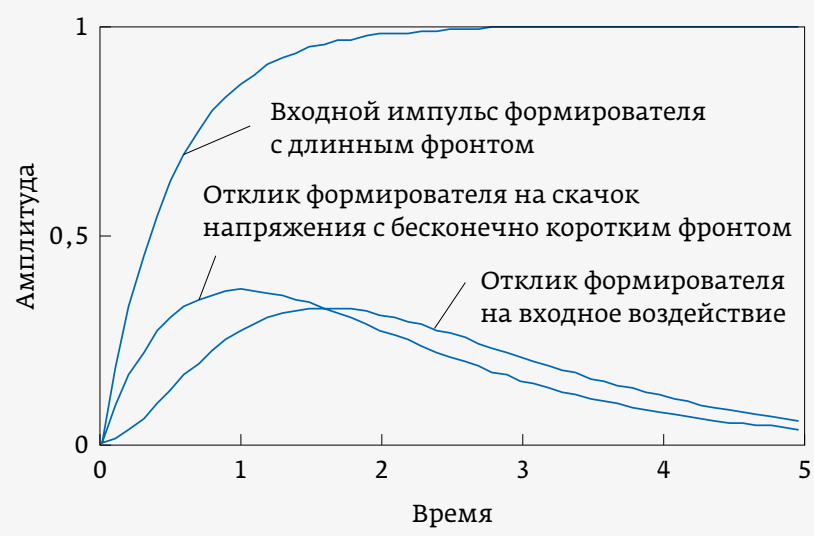

Рис. 15. Влияние длительности фронта входного импульса формирователя на отклик шейпера (время и амплитуды нормированы)

Корни 1 и р 2 квадратного трехчлена $1+\left(\tau_{b s}+\tau_{k}+\tau_{d}\right) \cdot s+$ $+\tau_{b s} \cdot \tau_{k} \cdot s^{2}$ из (23) всегда действительные и отрицательные (дискриминант равен $\left.\left(\tau_{b s}-\tau_{k}\right)^{2}+2\left(\tau_{b s}+\tau_{k}\right) \cdot \tau_{d}+\tau_{d}^{2} \geq 0\right)$. Это означает, что зчУ устойчив (полюса лежат в левой полуплоскости комплексной частоты). Проведя обратное преобразование Лапласа, из (23) получим отклик з4У во времени:

$$
U_{c s a}(t)=-\frac{C_{d}}{C_{f b}}\left[\begin{array}{c}
A \cdot \exp (-p 1 \cdot t)+B \cdot \exp (-p 2 \cdot t)+ \\
+C \cdot \exp \left(-\frac{t}{\tau_{f b}}\right)+D \cdot \exp \left(-\frac{t}{\tau_{p 1}}\right)
\end{array}\right],
$$

где 11 и р2 требуется применять с обратным знаком; A, B, C, D -коэффициенты разложения (23) на простые дроби.

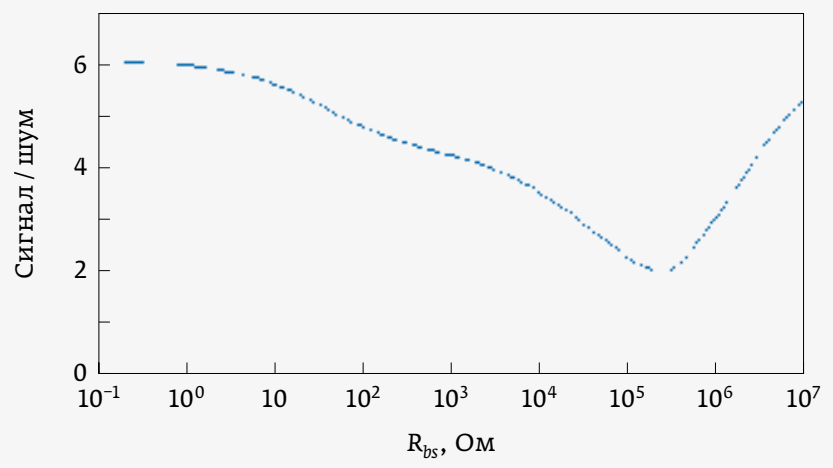

Рис. 16. Отношение сигнал/шум ЗЧу в зависимости от $\mathrm{R}_{b s}$ 
Форма сигналов на выходе зчУ с параметрами HES приведена на рис. 14.

Для нулевых $\mathrm{R}_{b s}$ и $\mathrm{R}_{k}$ сигнал представляет собой импульс с коротким фронтом и длинным экспоненциальным спадом (классический отклик 3чу). Последняя дробь в выражении (23) представляет собой фильтр второго порядка в области нижних частот. Это позволяет предположить, что при ненулевых сопротивлениях $\mathrm{R}_{k}$ и $\mathrm{R}_{b s}$ изменение формы импульса приведет к затягиванию фронта (снижению быстродействия) и падению его амплитуды.

Для высоких значений $\mathrm{R}_{b s}$ и $\mathrm{R}_{k}=0$ сигнал приобретает форму классического отклика зчу, но его амплитуда падает и определяется последовательным соединением $C_{d}$ и $C_{b s}$.

Затягивание фронта на входе шейпера изменяет его отклик на выходе (рис. 15). Представим входной сигнал формирователя CR-RC в виде напряжения с экспоненциальным фронтом $\tau_{f}$ :

$$
\mathrm{U}_{\mathrm{in}}(s)=\frac{1}{s}-\frac{\tau_{f}}{\left(1+\tau_{f} \cdot s\right)}
$$

Отклик формирователя будет равен:

$$
\mathrm{U}_{\text {out_sh }}(s)=\frac{1}{s} \cdot k_{\text {sh }}-\frac{\tau_{f}}{\left(1+\tau_{f} \cdot s\right)} \cdot k_{\text {sh }}=\frac{1}{s} \cdot k_{\text {sh }}-\frac{\tau_{f} \cdot s}{\left(1+\tau_{f} \cdot s\right)} \cdot k_{\text {sh }} \cdot \frac{1}{s},(26)
$$

где $k_{\text {sh }} \approx \mathrm{R}_{d} / \mathrm{R}_{i} ; \mathrm{R}_{d}$ и $\mathrm{R}_{\mathrm{i}}$ - сопротивления дифференцирующего и интегрирующего звеньев шейпера.

Увеличение длительности фронта на входе шейпера приводит к сдвигу максимума во времени и снижению быстродействия, а также к падению его амплитуды.

Из приведенных примеров видно, что источники шума независимы, различаются коэффициентами передачи в головном канале Сэ. Суммарное напряжение шу-

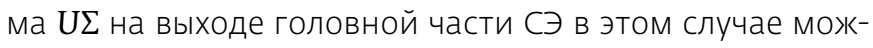
но выразить как:

$$
U_{\Sigma}=\sqrt{\sum_{1}^{n} U_{i}^{2}}
$$

где $U_{i}-$ напряжение шума каждого источника.

Исходя из результатов исследований в рамках проектов ZEUS-HES и ATIC можно утверждать, что паразитные элементы, такие как $R_{b s}$ и $R_{k}$ и другие [4], ухудшают соотношение сигнал/шум за счет увеличения шума и (или) снижения амплитуды сигнала. Для примера приведем суммарное соотношение сигнал / шум
зЧУ в зависимости от $\mathrm{R}_{b s}$ проекта ZEUS-HES для $\mathrm{R}_{k}=0$ (рис. 16).

Соотношение сигнал/шум (рис. 16) максимально для $\mathrm{R}_{\mathrm{bs}}=0$, когда сигнал максимален, а основной шум определяет последовательный шум зчу. Для больших значений $\mathrm{R}_{b s}$ соотношение сигнал / шум вновь начинает расти, поскольку АЧХ зчУ для сигнала опять становится широкополосной (см. рис. 14), но с пониженным коэффициентом усиления (кривая с $R_{k}=0, R_{b s}=10$ МОм), при этом полоса пропускания ЗЧУ для уменьшенного коэффициента усиления шире и, соответственно, шум выше.

$$
\therefore *
$$

Суммируя изложенное, можно отметить, что:

- источники напряжения смещения детекторов должны обладать низким уровнем шумов и помех, особенно в низкочастотной области, поскольку зчу для этого вида шумов является фильтром низкой частоты;

- контакты детекторов должны отличаться низкоомным сопротивлением, которое существенно ниже шумового сопротивления входного каскада, определяемого крутизной его первого транзистора;

- параллельный шум сопротивления разряда зчу имеет максимум, если постоянная времени цепи обратной связи равна времени формирования шейпера и может увеличивать суммарный шум;

- шумы детекторов могут включать специфические импульсные шумы в диапазоне 10-100 кГц, поэтому для подавления этих видов шумов предпочтительны шейперы с двойным дифференцированием, то есть с повышенной фильтрацией низкой частоты;

- затягивание фронта сигнала зчУ приводит к смещению максимума сигнала шейпера и падению амплитуды, что также снижает отношение сигнал / шум .

Результаты, представленные в статье, предполагается использовать в рамках реализации гранта РФФИ № 18-02-40047.

\section{ЛИТЕРАТУРА}

1. Воронин А. Структура каналов для считывающей электроники кремниевых детекторов // «ЭЛЕКТРОНИКА: Наука, Технология, Бизнес». 2019. № 2.

2. Spieler H. Electronics and data acquisition // Nuclear Instruments and Methods. 2012. V. AG66. P. 197-222.

3. Spieler H. Semiconductor Detector Systems // John Wiley Oxford University Press, New York U. S.A., 2005.

4. Воронин А. Моделирование кремниевых детекторов при разработке считывающей электроники. Часть 2 // «ЭЛЕКТРОНИКА: Наука, Технология, Бизнес». 2018. № 10.

5. Баскаков С. И. Радиотехнические цепи и сигналы / 3-е изд. - М.: Высшая школа, 2000. 